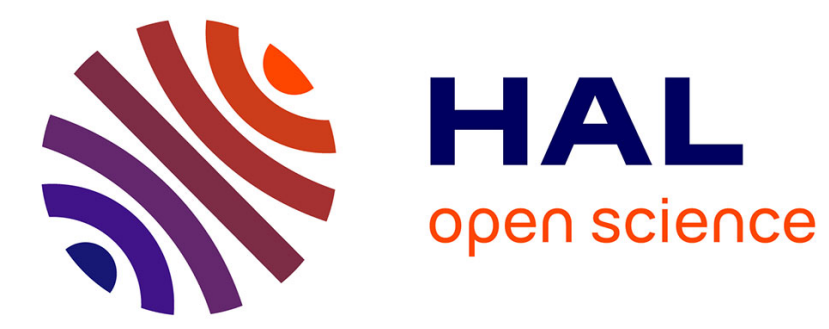

\title{
POLITIQUE DE LOGEMENT ET AUTONOMIE RÉSIDENTIELLE DE LA JEUNESSE EN ESPAGNE
}

Sandra Gaviria

\section{To cite this version:}

Sandra Gaviria. POLITIQUE DE LOGEMENT ET AUTONOMIE RÉSIDENTIELLE DE LA JEUNESSE EN ESPAGNE. Informations sociales, 2011, 10.3917/inso.165.0146 • hal-02385981

\section{HAL Id: hal-02385981 \\ https://hal.science/hal-02385981}

Submitted on 29 Nov 2019

HAL is a multi-disciplinary open access archive for the deposit and dissemination of scientific research documents, whether they are published or not. The documents may come from teaching and research institutions in France or abroad, or from public or private research centers.
L'archive ouverte pluridisciplinaire HAL, est destinée au dépôt et à la diffusion de documents scientifiques de niveau recherche, publiés ou non, émanant des établissements d'enseignement et de recherche français ou étrangers, des laboratoires publics ou privés. 


\section{POLITIQUE DE LOGEMENT ET AUTONOMIE RÉSIDENTIELLE DE LA JEUNESSE EN ESPAGNE

\author{
Sandra Gaviria
}

Caisse nationale d'allocations familiales (CNAF) | «Informations sociales »

2011/3 n 165-166 | pages 146 à 154

ISSN 0046-9459

Article disponible en ligne à l'adresse :

http://www.cairn.info/revue-informations-sociales-2011-3-page-146.htm

\section{Pour citer cet article :}

Sandra Gaviria, " Politique de logement et autonomie résidentielle de la jeunesse en Espagne », Informations sociales 2011/3 ( $\left.\mathrm{n}^{\circ} 165-166\right)$, p. 146-154.

Distribution électronique Cairn.info pour Caisse nationale d'allocations familiales (CNAF).

(C) Caisse nationale d'allocations familiales (CNAF). Tous droits réservés pour tous pays.

La reproduction ou représentation de cet article, notamment par photocopie, n'est autorisée que dans les limites des conditions générales d'utilisation du site ou, le cas échéant, des conditions générales de la licence souscrite par votre établissement. Toute autre reproduction ou représentation, en tout ou partie, sous quelque forme et de quelque manière que ce soit, est interdite sauf accord préalable et écrit de l'éditeur, en dehors des cas prévus par la législation en vigueur en France. Il est précisé que son stockage dans une base de données est également interdit. 


\section{Politique de logement et autonomie résidentielle de la jeunesse en Espagne}

Sandra Gaviria - sociologue

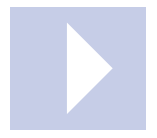

Les jeunes Espagnols quittent le domicile parental plus tardivement que les autres jeunes Européens. Si les médias et les politiques ont voulu y voir l'effet $d^{\prime}$ un " problème $d u$ logement pour les jeunes ", il s'agit en fait d'une tradition bien ancrée. Et quand ils partent de chez leurs parents, leur choix massif d'accéder à la propriété s'inscrit aussi dans la norme, l'Espagne comptant l'un des plus forts taux de propriétaires en Europe.

Il existe en Espagne un modèle fortement ancré de départ tardif des jeunes du domicile parental, généralement suite au mariage et à l'achat concomitant d'un logement. Ce type de trajectoire a toujours été valorisé par l'État au travers de sa politique de logement. Pourtant, les médias véhiculent constamment l'idée du grand problème de la jeunesse, qui serait celui de son incapacité à quitter le domicile familial pour aller vivre ailleurs. On peut lire dans les médias : «Le logement, un problème jeune » (1), ou encore «Le logement, un problème autant pour les jeunes que pour les personnes âgées » (2). Les politiques intègrent régulièrement le problème de l'accès au logement des jeunes dans leurs discours. À titre d'exemple, en 2006, aux élections gouvernementales de Catalogne, tous les partis politiques faisaient du logement pour les jeunes l'une de leurs priorités et mettaient en place des propositions électorales en la matière. Toutefois, aujourd'hui, compte tenu de la crise économique que traverse le pays, ceci est en train d'évoluer et les principaux sujets de débat sont plutôt le chômage et les restrictions budgétaires. Ainsi, aux élections de Catalogne de novembre 2010, le logement des jeunes n'était plus une priorité dans les discours électoraux; seuls les programmes du Partits del Socialistes de Catalunya (PSC) et du Partido Popular (PP) y faisaient clairement référence. 
Pour comprendre la réalité espagnole, nous montrerons (3) dans un premier temps comment la politique de la jeunesse est apparue tardivement, ainsi que l'intérêt des gouvernements pour le logement. Dans un deuxième temps, que la politique de logement envers les jeunes a majoritairement favorisé l'accès à la propriété, les solutions de location ayant peu de succès. Enfin, dans un troisième temps, nous verrons que les intéressés souhaitent majoritairement devenir, comme leurs amis, leurs parents et leurs grands-parents, des propriétaires en s'installant et en se projetant de façon durable.

\section{Historique de la politique de la jeunesse}

Pour comprendre la politique de la jeunesse en Espagne, il est intéressant de faire un détour sur les caractéristiques de cette classe d'âge selon les périodes historiques. Entre les années 1940 et les années 1960, les jeunes sont dépendants de la famille et invisibles dans la sphère publique. Au début des années 1960 et jusqu’à fin des années 1970, les étudiants entrent en conflit avec le système ; ils manifestent contre la dictature et acquièrent une visibilité absente jusqu'alors. Les lois deviennent plus fermes à leur encontre, avec une volonté de contrôle. Ainsi, par exemple, en 1973 la majorité pénale est établie à 16 ans. Avec l'avènement de la démocratie à la fin des années 1970 commence une phase d'identification de problèmes qui concernent les jeunes. La politique de la jeunesse en Espagne est apparue tardivement par rapport à d'autres pays européens. Elle n'existait pas encore réellement lorsque la Constitution est approuvée, en

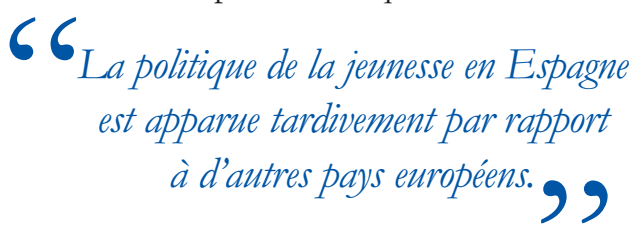

1978. Il n'y avait ni tissu social ni tradition sur lesquels la politique pouvait prendre appui (Comas Arnau, 2007). Son origine remonte à l'instauration des premières municipalités démocratiques et à la naissance de plans locaux de jeunesse et des conseils municipaux des jeunes.

Au niveau national, dans les années 1980, se créent les communautés autonomes (CCAA) et l'État transfère les compétences dans le domaine de la jeunesse à dix-sept d'entre elles, ne conservant que les compétences de l'Injuve, l'Institut de la jeunesse (chargé des échanges internationaux, études et publications ainsi que de l'encouragement des pratiques artistiques).

La répartition entre l'État, les provinces et les communes constitue une difficulté pour appréhender la politique de la jeunesse. Dans les années 1980-1990 s'élaborent les plans intégraux de jeunesse ou Plan Joven. Ils ont l'ambition d'intervenir sur l'ensemble des domaines de la vie des jeunes et requièrent l'intervention des différents services de l'administration municipale. Ils ont un succès relatif car "les services jeunesse n'ont conservé qu'un rôle secondaire dans l'administration locale»(Garcia et Merino, 2006, p. 74). 
Les préoccupations pour le logement et l'emploi apparaissent au moment où la jeunesse est perçue comme un problème, au cours des années 1990 (Comas Arnau, 2007). L'âge limite de la jeunesse est fixé à 35 ans à partir de cette époque, contre 25 ans dans les années 1970, pour tenir compte du retard dans le calendrier de l'accès à l'indépendance. Le logement a fait tardivement partie des inquiétudes de la politique de la jeunesse au niveau national. Ce n'est que dans le plan jeunesse 1994-1997 que la préoccupation du logement apparait. Dans le plan interministériel de jeunesse de 2005-2008, l'emploi et le logement constituent un des six axes stratégiques (Comas Arnau, 2007). L'objectif de ce plan est de faire collaborer les différentes administrations. Ainsi, quatorze départements ministériels sont concernés. Les mesures prévues consistent à faciliter l'accès à la location et à la propriété (4).

\section{Une politique du logement basée sur l'accès à la propriété}

Depuis l'époque franquiste, la politique du logement a toujours favorisé l'accès à la propriété de la population, soutenue par l'idée qu'un pays de propriétaires était un pays de patriotes. L'idée subsiste encore aujourd'hui, parmi les experts et les politiques (5), que la propriété a des vertus de cohésion sociale. Ainsi, les familles s'attachent à leur ville, leur quartier, leur immeuble et leur cage d'escalier. Les liens sociaux créés avec les voisins sont plus stables dans le temps. Les propriétaires vont être plus soigneux afin que leur logement ne se dévalorise pas. Les associations de voisinage, par exemple, ont eu et continuent à avoir un rôle important dans la lutte contre la drogue, comme dans les années 1980 avec les problèmes d'héroïne (6).

La politique de logement a certes toujours favorisé la propriété des personnes installées et des jeunes au moment de la création d'une famille, mais pas pour une population célibataire étudiante. L'Espagne est aujourd'hui l'un des pays européens avec le taux le plus élevé de logements en propriété, à savoir $86 \%$ (7). Le parc de logements en location est ainsi modeste, avec $14 \%$ des logements (12\% privé et $2 \%$ social), contrairement à d'autres pays européens comme la France où il est de $46 \%(21 \%$ privé, $17 \%$ social HLM, et $8 \%$ autres cédés par des entreprises gratuitement, 2001) (8).

La propriété a toujours été favorisée pour l'ensemble de la population, mais qu'en est-il de la jeunesse ? Jusqu'à très récemment, il n’y avait pas eu de vraie politique de logement en faveur des jeunes, pas plus qu'en faveur des locataires. Ces dernières années, compte tenu des difficultés grandissantes dans l'émancipation, les premières mesures ont été mises en place par le gouvernement Zapatero. Depuis 2003, l'Observatoire du logement jeune (9) réalise des enquêtes régulières afin de mieux saisir les comportements et le marché immobilier. Toutefois, il faut comprendre que les 
budgets mis à disposition pour le logement et la jeunesse sont très limités et, de fait, les mesures restent plus symboliques que réellement efficaces. Quelques mesures pour les jeunes ont favorisé la location, mais sans grand succès. À partir de 1995 (Gaviria, 2005), certaines communautés autonomes et villes se sont préoccupées du problème de logement pour les jeunes. Saragosse ${ }^{(10)}$, par exemple, a mis en place une politique de logements en location, subventionnés pendant deux ans par l'État, avec la bolsa vivienda (allocation

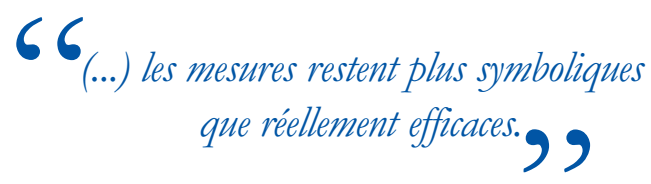
logement). En fait, il n'y a eu pratiquement pas de demande de la part des jeunes au cours de cette expérience. Le rapport (11) établi par la suite par l'architecte en chef du Bureau municipal du Plan général d'aménagement urbain de Saragosse, pour déterminer les lignes conductrices du Plan Joven en faveur de la jeunesse, a proposé une politique pour attirer les jeunes vers la location des logements rénovés dans la vieille ville. Le but était de modifier leurs comportements et les options d'accès à des logements neufs en propriété, et d'essayer en même temps de comprendre les raisons de leur rejet de la location. Il note que les populations comprises entre 25 et 35 ans ou entre 35 et 40 ans restent prisonnières de l'achat de leur logement et peuvent parfois y consacrer jusqu'à $50 \%$ de leurs revenus pour le payer. À ses yeux, cela signifie qu'ils consacrent trop de ressources à leur logement, ce qui les rend extrêmement dépendants de leur situation économique, et qu'il leur reste peu d'argent pour des projets personnels d'envergure.

Au cours de ces dernières années, il y a eu davantage de mesures favorisant la location, mais sans plus de succès (Jurado, 2006). Seulement $11 \%$ des espagnols vivent dans une location. Il s'agit de jeunes, divorcés, mères célibataires, concubins et immigrés. En 2007 (12), la ministre du Logement María Antonia Trujillo avait proposé de créer en propriété ou en location les minipisos (minilogements) à destination des jeunes (13). Ces logements de 30 mètres carrés devaient permettre d'avancer le départ de chez les parents. Cela a généré une levée de boucliers pendant des mois, les médias qualifiant ces mesures de «barbares» et «indignes »comment la ministre pouvait-elle proposer des logements si petits? Les médias et la population se sont faits les porte-parole du modèle commun d'accès à l'indépendance : un logement en propriété, de taille familiale, puisque souvent le départ arrive au moment du mariage. La dernière mesure phare, établie en 2008 au niveau national, est la « Rente pour l'émancipation». Elle consiste en une aide à la location de 210 euros, pendant quatre ans, pour les 22-30 ans qui travaillent depuis deux ans et qui ont des revenus inférieurs à 22000 euros (14) par an (15). À noter que le salaire moyen des 18-34 ans est de 15263 euros, les revenus moyens d'un foyer jeune de 22526 euros. De plus, cette rente s'adresse 
aux actifs de plus de 22 ans et pas aux étudiants. En fait, l'État n'a pas aidé les moins préparés et les plus démunis, mais ceux qui avaient déjà un emploi. Au total, en 2010 (16), seules 196898 aides ont été distribuées, c'est-à-dire $4 \%$ des jeunes émancipés. Les taux les plus élevés correspondent à la Communauté autonome de Catalogne et à celle de Madrid, représentant $34 \%$ de l'ensemble. On peut penser que cette rente consiste plus en un complément de salaire des mileuristas (les «mileuristes » sont des jeunes actifs qui ne gagnent que 1000 euros par mois et sont restreints dans leur capacité d'indépendance) que dans une aide au départ vers son propre logement.

Enfin, compte tenu des difficultés existantes pour certains publics à accéder à la propriété, des mesures d'accès différé à la propriété ont été mises en place. Elles permettent aux individus de payer un loyer et, après quelques années, s'ils sont en mesure d'accéder à ladite propriété, ces loyers sont déduits du prix total du logement. Il faut par ailleurs signaler qu'il n'y a jamais eu de dispositifs de logement universitaire ; quelques programmes mineurs se mettent en place depuis peu.

\section{Un jeune propriétaire est un adulte intégré}

Les Espagnols restent donc tardivement au domicile familial. Le modèle de construction de soi pour devenir adulte se fonde sur une forte identité commune avec la famille. Il est établi en Espagne que vivre ensemble favorise ce type de construction de soi, à travers le partage de moments et d'expériences.

Le départ, qui se fait souvent au moment du mariage, est un départ définitif sans idée de retour possible. Il a lieu quand les conditions économiques et matérielles sont réunies. Pourtant, les discours politiques continuent d'avancer des explications matérielles et le problème du logement pour justifier la permanence tardive au domicile. À nos yeux, ces motifs peuvent contribuer à expliquer les retards dans les calendriers de départ, mais en aucun cas le choix de la propriété. Ainsi, lorsque les jeunes ont recours à la location, il ne s'agit généralement que d'une période de transition. Ils souhaitent des conditions de logement pas forcément en lien avec leur situation présente (Consejo económico y social - CES, 2002), mais conformes à leur vie future et dans une perspective pérenne.

En 2009, l'Espagne compte onze millions de jeunes (17). Chez les 18-34 ans, $54 \%$ habitent chez leurs parents. Parmi ceux qui travaillent, $63 \%$ ont un CDI et $37 \%$ un CDD. Le taux de chômage est de $25 \%$. Le départ se fait lorsque les couples ont une ou deux voitures et chacun un emploi en CDI ou CDD, ainsi qu'un compte épargne logement. Ils préfèrent acquérir un logement neuf et souhaitent l'aménager au maximum avec des meubles neufs. Ce modèle est soutenu par les familles qui souvent 
« retiennent» les enfants pour leur permettre d'économiser davantage et ainsi de réaliser leur souhait. Les enquêtes montrent également que la probabilité d'avoir un enfant en Espagne est étroitement liée au fait d'être ou non propriétaire, contrairement à d'autres pays européens (Gonzalez et Jurado, 2006). Socialement, un jeune est considéré comme intégré et devenu adulte lorsqu'il suit le modèle défini comme bon, celui qui consiste à avoir un emploi, se mettre en couple et accéder à la propriété. Ce dernier aspect constitue un acte de responsabilité fort car cela signifie que ce jeune adulte a travaillé quelques années, qu'il a su économiser et être prévoyant, puis qu'il s'engage dans un projet à très long terme. Il entre dans la norme sociale.

Plusieurs éléments sont significatifs du fait que la propriété est le modèle existant souhaité par les jeunes. Dans les enquêtes, ils mettent en valeur leur envie d'accès à la propriété et leur rejet de la location. Chez les jeunes de 18 à 34 ans qui habitent chez leurs parents (Injuve, 2006), ceux qui comptent partir dans les trois prochaines années expriment les choix suivants : $90 \%$ préfèrent un logement en propriété et 7,4\% en location. En Catalogne, en 2001 (Albarat, Gavaldá et Vilá, 2008), 68 \% des foyers de jeunes sont propriétaires $(14,5 \%$ d'entre eux ont fini de payer leur logement et 53,3\% doivent encore en payer une partie), $26 \%$ sont locataires et $6 \%$ relèvent d'autres catégories. La moitié des jeunes qui partent de chez leurs parents pour fonder un foyer accèdent à la propriété et, parmi ceux qui sont en location, $50 \%$ accè-

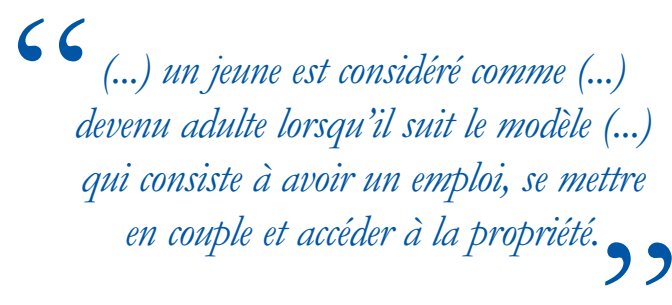
dent à la propriété au bout de deux ans (Leal, 2010).

La décohabitation tardive des jeunes Espagnols est attribuée dans les médias au prix élevé des logements en location ou en propriété qui les empêcherait de partir. Les difficultés pour accéder à un logement sont effectivement importantes, car il y a eu une augmentation des prix tout au long des années 1980 (Leal Maldonado, 1997). Ainsi, au cours de certaines périodes, comme celle de 1985 à 1990, il y a eu une amélioration de l'emploi, mais le marché du logement n'a pas pu s'adapter avec rapidité, la conséquence étant l'augmentation de la demande et donc une augmentation des prix (idem).

Entre 1996 et 2005, période d'essor économique et de faible chômage, il n'y a pas eu de grands changements dans les chiffres à cause des prix élevés des logements. Aujourd'hui, les prix ont diminué (d'environ $20 \%$ ) mais les jeunes n'ont pas les moyens d'acheter, du fait de la difficulté d'accès à l'emploi et du resserrement des conditions de crédit par les banques. Ceci a pour conséquence un marché bloqué, et ce pour de nombreuses années certainement. 
La Rente pour l'émancipation mise en place par l'État est une fausse réponse à un faux diagnostic : croire que les jeunes ne partaient pas en raison du seul manque de moyens pour accéder à la location. Il y a une médiatisation de la difficulté pour les jeunes d'accéder à un logement et de leur départ tardif comparé à leurs voisins européens, mais politiquement rien n'est mis en place massivement pour un accès à la location pendant la période étudiante. Le modèle développé par les politiques publiques est celui d'un départ tardif, en propriété, dans un logement de taille familiale. Il y a donc un décalage entre les discours communément admis et médiatisés et ce qui est fait politiquement.

Les politiques favorisant la location ont été souvent confrontées à la résistance de la population. Les jeunes continuent à souhaiter accéder à la propriété et la considèrent comme essentielle. Lorsqu'ils deviennent locataires, c'est de manière transitoire et parce qu'ils n'ont pas eu d'autre choix. Finalement, le modèle souhaité par les politiques, les jeunes et les familles est celui d'un départ après la période étudiante pour accéder à la propriété. Compte tenu de la crise actuelle, le modèle va être difficile à maintenir dans les prochaines années et le prolongement de la jeunesse au sein du domicile familial risque de s'accentuer fortement (18).

\section{Notes}

1 - El Mundo, 18 juillet 1995

2 - El Mundo, 10 août 2007.

3 - Ces résultats sont issus d'une enquête de terrain auprès de 30 jeunes espagnols de 25 à 30 ans, $d^{\prime}$ une revue de la bibliographie sociologique existante sur le sujet ainsi que de discussions avec des politiques sur la question du logement des jeunes.

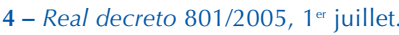

5 - El País, 15 décembre 2008

6 - Madridiario.es, "Los vecinos de Vallecas crean un grupo en Facebook para denunciar los "taxis de la droga" », 24 octobre 2010 .

7 - Europa.eu, Annuaire 2008, moyenne européenne $63 \%$, France $58 \%$.

8 - Ministerio de Vivienda.

9 - Observatorio joven de vivienda.

10 - Saragosse est considérée comme une ville représentative de la moyenne du pays.

11 - Informe preliminar para la elaboración del Plan Joven de Zaragoza, 1996.

12 - El País, 7 juillet 2007 
13 - Luis Doncel, « La ministra de los minipisos », El País, 7 juillet 2007.

14 - Un salaire de 22000 euros est considéré comme élevé en Espagne.

15 - L'État participe au financement de la caution de 600 euros et aux frais de gestion de 120 euros.

16 - Au mois de mars.

17 - Observatorio joven de la vivienda. Dernier trimestre 2009.

18 - La Rente pour l'émancipation sera sûrement supprimée dans les prochains mois, comme beaucoup d'autres aides.

\section{Bibliographie}

- Albarat A., Gavaldá J. et Vilá G., 2008, "Los jóvenes y la vivienda », in Bellet C., Ganau J. et Llop J.-M. (dir.), Vivienda y sociedad, Lleida, Milenio, p. 491-516.

- Comas Arnau D., 2007, Las políticas de juventud en la España democrática, Madrid, Injuve.

- Comisión interministerial para la juventud y la infancia, 2006, Plan interministerial de juventud 2005-2008, Madrid, Injuve, Ministerio de trabajo y asuntos sociales.

- Consejo económico y social (CES), 2002, La emancipación de los jóvenes y la situación de la vivienda en España, Madrid.

- Consejo de la juventud de España, 2010, Observatorio de la vivienda joven, $4^{\mathrm{e}}$ trimestre 2009.

- Freire S., 2006, Los mileuristas : cuerpo, alma y mente de la generación de Ios 1000 euros, Barcelone, Ariel.

- Garcia M. et Merino R., 2006, "Espagne, théorie et pratique des politiques de jeunesse », Agora, ${ }^{\circ}$ 42, p. 72-85.

- Gaviria S., 2005, Quitter ses parents ; une comparaison franco-espagnole, Rennes, Presses universitaires de Rennes, (Pur), coll. "Le Sens social».

- Gonzalez M.-J. et Jurado T., 2006, "Remaining childless in Affluent Economies : a comparaison of France, West Germany, Italy and Spain, 1994-2001», European Journal of population, vol. 22, n 4, p. 317-352.

- Instituto de la Juventud (Injuve), 2006, " Juventud y vivienda. Sondeo de opinión y situación de la gente joven $2005 »$, ministère de la Santé, de la Politique sociale et de l'Égalité, Juventud y vivienda, tablas de resultados y conclusiones.

- Jurado T., 2006, «El creciente dinamismo familiar frente a la inflexibilidad del modelo de vivienda español », Cuadernos de información económica, $\mathrm{n}^{\circ} 193$, juillet-août, p. 117-126.

- Jurado Guerrero T., 1999, "Why do French people leave home earlier than Spaniards ? The role of employment, housing and social policies ", thèse de doctorat (Crouch C., directeur, et Breen R., codirecteur), European University Institute, Italie. 
- Leal J., 2010, "Stratégie résidentielle des jeunes foyers et formation d'espaces sociaux urbains en Espagne », in Authier J.- Y., Bonvalet C. et Lévy J.-P. (dir.), Élire domicile : la construction sociale des choix résidentiels, Lyon, Presses universitaires de Lyon (Pul), p. 315-332.

- Leal Maldonado J., 2000, "Comportamientos residenciales y necesidades de vivienda ", in Taltavull P. (dir.), Vivienda y familia, Madrid, Fundación Argentaria y Visor Dis, p. 365-385; 1997, "Emancipación y vivienda », in Vergés R. (dir.), La edad de emancipación de los jóvenes, Barcelone, Centro de cultura contemporània, p. 113-124.

- Raya Vilchez J.-M., 2007, Emancipación y vivienda : una identidad con tres componentes, ACE : Arquitectura, Ciudad y Entorno, année II, $n^{\circ} 5$, octobre.

- Sanchez-Martinez M.-T., 2002, La política de vivienda en España. Análisis de sus efectos redistributivos, Grenade, Université de Grenade.

- Trilla C., 2001, La política de vivienda en una perspectiva comparada, Barcelone, La Caixa. 